\title{
Modelling Ag-particle activation and growth in a TSI WCPC model 3785
}

\author{
F. Stratmann ${ }^{1,2}$, E. Herrmann ${ }^{1}$, T. Petäjä ${ }^{1}$, and M. Kulmala ${ }^{1}$ \\ ${ }^{1}$ Department of Physics, P.O. Box 64, 00014 University of Helsinki, Finland \\ ${ }^{2}$ Leibniz Institute for Tropospheric Research (IfT), Permoserstrasse 15, 04318 Leipzig, Germany
}

Received: 6 August 2009 - Published in Atmos. Meas. Tech. Discuss.: 25 September 2009

Revised: 17 February 2010 - Accepted: 22 February 2010 - Published: 25 February 2010

\begin{abstract}
In this work, we modelled activation and growth of silver particles in the water-operated TSI model 3785 water condensation particle counter (WCPC). Our objective was to investigate theoretically how various effects influence the counting efficiency of this CPC. Coupled fluid and particle dynamic processes were modelled with the computational fluid dynamics (CFD) code FLUENT in combination with the Fine Particle Model (FPM) to obtain profiles of temperature, vapour concentration, nucleation rate, and particle size. We found that the counting efficiency of the TSI 3785 for small particles might be affected by the presence of larger particles. Moreover, homogeneous nucleation processes can significantly influence counting efficiency.
\end{abstract}

\section{Introduction}

Condensation Particle Counters (CPCs) measure number concentration of aerosol particles. These instruments can detect sub-micrometer particles that cannot be perceived by optical counters (McMurry, 2000). The operational principle of a CPC is generally a multi-step process. First, the aerosol laden air is saturated with a vapour, and then the mixture is brought into supersaturated conditions with respect to this vapour, which leads to activation and growth of the particles to optically detectable sizes. The supersaturation can be created by adiabatic expansion (e.g. Kürten et al., 2005), conductive cooling (e.g. Sem, 2002), thermal diffusion (Hering and Stolzenburg, 2005; Hering et al., 2005), and mixing of cool and warm saturated air flows (Sgro and Fernandez de la Mora, 2004).

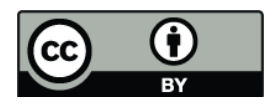

Correspondence to: E. Herrmann (erik.herrmann@helsinki.fi)
Hering and Stolzenburg (2005) introduced a method to generate supersaturation by simultaneous diffusion of water and heat. Inside a wet-walled growth tube, condensational growth of the particles is initiated by heating the aerosol flow. Due to the fact that mass diffusivity of water in air is higher than the thermal diffusivity of humid air, a supersaturated area is generated inside the growth tube. Thus, the created supersaturated conditions at the centreline allow the condensational growth of the aerosol particles and their subsequent detection (Hering et al., 2005). Petäjä et al. (2006) showed that the detection efficiency for silver particles of this Water-based Condensation Particle Counter (WCPC) depends on the temperature difference between the saturator and the growth tube.

The experiments in Petäjä et al. (2006) indicated that the solubility of the sampled particles affects the detection efficiency of the water-based CPC considerably. The cut-off sizes were $5.8 \mathrm{~nm}$ and $3.6 \mathrm{~nm}$ for silver and sodium chloride particles, respectively. Thus, the amount of supersaturation, the physico-chemical properties of the working fluid, and the sampled particles influence the activation probability inside the CPC and thus determine the detection efficiency of the instrument (e.g. Stolzenburg and McMurry, 1991; Kulmala et al., 2007; Iida et al., 2009). The activation of the particles soluble to the working fluid can be described with Köhler theory (e.g. Kulmala et al., 2004) whereas the activation of insoluble particles can be modelled by a heterogeneous nucleation theory (e.g. Lazaridis et al., 1992).

The objective of this study is to examine a TSI model 3785 Water Condensation Particle Counter (WCPC) in a detailed modelling framework. For describing the coupled fluid and particle dynamical processes taking place inside the WCPC, a Computational Fluid Dynamics Code FLUENT (Fluent, 2005) together with a Fine Particle Model (FPM) (particle dynamics, 2005) were used. Utilizing this combined fluid and particle dynamical model, the effects of heterogeneous

Published by Copernicus Publications on behalf of the European Geosciences Union. 
and homogeneous nucleation on the counting efficiency of the instrument were addressed. Following earlier investigations by Stolzenburg and McMurry (1991) and Saros et al. (1996) on the effect of particle number concentration on vapour depletion and thus counting efficiency, the effect was now examined for the case of the TSI 3785 WCPC with the help of a sophisticated model.

\section{Methods}

\subsection{Description of the TSI WCPC 3785}

The TSI 3785 condensation particle counter (Hering et al., 2005; Hering and Stolzenburg, 2005) is a laminar flow instrument the operation principle of which is based on the condensation of water on aerosol particles to enlarge these particles to optically detectable sizes. Figure 1 shows a simplified schematic diagram of the instrument. The components central to this study are the saturator and the growth tube. The inner walls of these tubes are temperature controlled and lined with a single wick made of a hydrophilic porous material which is kept wetted by a water bath. The default temperature values for the saturator and growth tube walls are $293.15 \mathrm{~K}$ and $333.15 \mathrm{~K}$, respectively. Due to the wetted walls, the saturation ratio is 1 at the inner walls of saturator and growth tube. Aerosol enters the saturator through an inlet at a flow rate of $1.0351 \mathrm{~min}^{-1}$ and is pre-conditioned by heat and water vapour diffusing from the saturator wall. The actual temperature and saturation ratio achieved depends on the conditions at the saturator inlet (in these investigations set to $293.15 \mathrm{~K}$ and $0 \%$ relative humidity). Upon entering the growth tube, the wall temperature is increased. For water vapour in air, mass diffusion is faster than heat diffusion which leads to supersaturation inside the tube. Depending on the actual supersaturation $S$, aerosol particles from a certain size on will be activated and grow in size. Large enough particles will be detected and counted by an optical particle counter (OPC) downstream of the growth tube.

\subsection{Modelling}

For describing the coupled fluid and particle dynamical processes taking place inside the CPC the Computational Fluid Dynamics Code FLUENT (Fluent, 2005) together with the Fine Particle Model (FPM) (particle dynamics, 2005) were used. Utilizing this combined fluid and particle dynamical model, from now on called FLUENT/FPM, the following processes were solved for:

- fluid momentum (assuming a parabolic velocity profile)

- vapour mass transfer (accounting for multi-component and thermal diffusion, vapour source at the wall and vapour sink due to condensation of vapour to the particles and associated latent heat release)

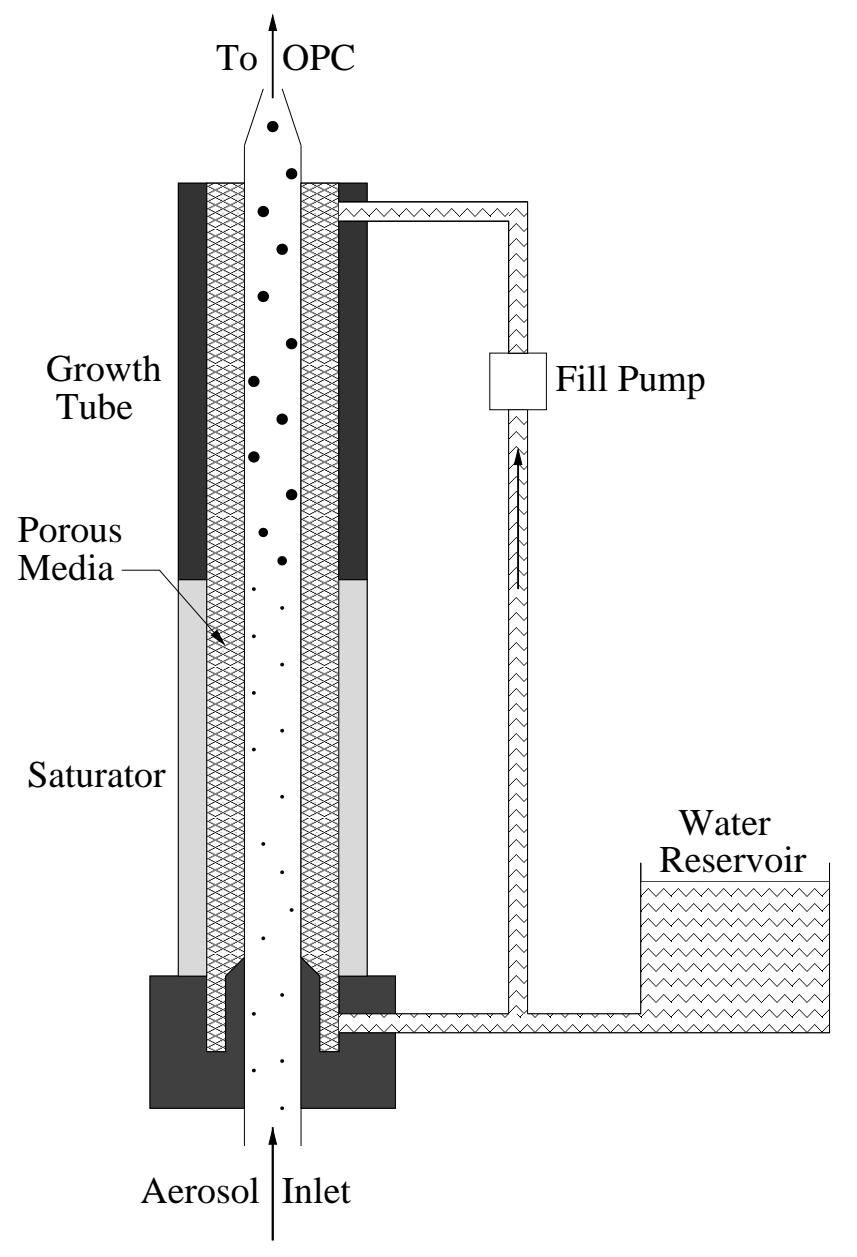

Fig. 1. A schematic diagram of a TSI 3785 CPC.

- heat transfer

- particle transport due to convection

- homogeneous (Girshik et al., 1990, used without correction) and heterogeneous nucleation (Fletcher, 1958; Lazaridis et al., 1991)

- particle transformation due to condensation/evaporation (Barrett and Clement, 1988)

For the above model setup and assumptions, the governing equations take the following form:

\subsubsection{Vapour mass transfer}

$$
\begin{aligned}
\nabla \cdot\left(\rho \mathbf{u} \omega_{v}\right) & =-\nabla \cdot \mathbf{j}_{v}+S_{v} \\
\mathbf{j}_{v} & =-\rho D_{v} \nabla \omega_{v}-\rho D_{v} \alpha_{v, g}\left(1-\omega_{v}\right) \omega_{v} \nabla \ln T
\end{aligned}
$$

Here $\mathbf{j}_{v}$ is the mass flux of vapour relative to the mass average velocity $\mathbf{u}, \rho$ is the density and $T$ is the absolute temperature of the vapour-carrier-gas mixture; $\omega_{v}, D_{v}$, and $\alpha_{v, g}$ are the 
vapour mass fraction, the binary vapour diffusion coefficient, and the thermal diffusion factor, respectively; and $S_{v}$ stands for the vapour sink due to condensation onto the particles.

\subsubsection{Heat transfer}

$$
\begin{aligned}
\nabla \cdot(\rho \mathbf{u} h)= & -\nabla \cdot \mathbf{q}+S_{h} \\
\mathbf{q}= & -\rho \alpha \nabla h-\rho\left(\alpha \nabla \omega_{v}+\mathbf{j}_{v}\right)\left(h_{v}-h_{g}\right) \\
& +\alpha_{v, g} k T \frac{M}{M_{v}+M_{g}} \mathbf{j}_{v}
\end{aligned}
$$

Here $\mathbf{q}$ is the heat flux, $h$ is the specific enthalpy, $\alpha=k /\left(\rho c_{p}\right)$ is the thermal diffusivity, $k$ is the heat conductivity, $c_{p}$ is the specific heat capacity; $M$ is the molar weight of the vapourgas mixture, $h_{v}$ and $h_{g}$ are the specific enthalpies, $M_{v}$ and $M_{g}$ are the molar weights of the vapour and the carrier gas, respectively; and $S_{h}=L_{v} S_{V}$ represents the heat source, that is, the latent heat ( $L_{v}$ is the vapour heat of vaporization) released by the vapour condensing onto the particles.

\subsubsection{Particle transport and transformation}

During the theoretical calculations, uni- and/or bi-modal moving monodisperse or lognormal particle size distributions were considered. Here, for convenience and simplicity, only the equations for the moving monodisperse model, i.e. the equations describing the conservation of mode $j$ particle number $N_{p, j}[\# / \mathrm{kg}$ ] and species $i$ mass concentrations $M_{p, i, j}[\mathrm{~kg} i / \mathrm{kg}]$, are given. For a detailed description of the polydisperse model, the reader is referred to FPM user's guide (particle dynamics, 2005).

$\nabla \cdot\left(\rho \mathbf{u} N_{p, j}\right)=\nabla \cdot \rho D_{p, j} \nabla N_{p, j}+S_{N p, j}$

$\nabla \cdot\left(\rho \mathbf{u} M_{p, j}\right)=\nabla \cdot \rho D_{p, j} \nabla M_{p, j}+S_{M p, i, j}+N_{p, j} \cdot \frac{\partial m_{p, i, j}}{\partial t}$

Here, $i$ denotes the different substances in the particle/droplet phase (silver and water), $S_{N p, j}$ and $S_{M p, i, j}$ are number and mass sources due to homogeneous nucleation, respectively, and $\partial m_{p, i} / \partial t$ corresponds to the single particle condensational growth rate (see below).

\subsubsection{Heterogeneous nucleation}

In a heterogeneous nucleation process, small nuclei form on pre-existing aerosol particles. Since we study the condensation of water vapour on silver particles, we need to consider only non-soluble particles. Classical nucleation theory gives the critical cluster formation rate for the heterogeneous nucleation as (Fletcher, 1958)

$$
J_{\text {het }}=K \exp \left(\frac{-\Delta G_{\text {het }}^{*}}{k T}\right)
$$

where $K$ is a kinetic pre-factor and $\Delta G_{\text {het }}^{*}$ the formation energy of the critical heterogeneous nucleus. The formation energies of the heterogeneous and homogeneous nuclei are connected by

$$
\Delta G_{\mathrm{het}}^{*}=f(m, X) \Delta G_{\mathrm{hom}}^{*}
$$

where

$$
\begin{aligned}
f(m, X) & =1+\left(\frac{1-m X}{g}\right)^{3} \\
& +X^{3}\left(2-3\left(\frac{X-m}{g}\right)+\left(\frac{X-m}{g}\right)^{3}\right) \\
& +3 m X^{2}\left(\frac{X-m}{g}-1\right)
\end{aligned}
$$

with

$g=\sqrt{1+X^{2}-2 m X}$

is a geometric factor that depends on the ratio of dry particle radius and critical cluster size through $X=R_{\text {dry }} / r^{*}$ and on the macroscopic contact angle $\theta$ through $m=\cos \theta$ (Lazaridis et al., 1992). $\theta=0$ represents a perfectly wettable surface whereas $\theta=180^{\circ}$ corresponds to a completely unwettable surface. This indicates homogeneous nucleation and accordingly $f$ becomes 1 in this case.

From the heterogeneous rate the heterogeneous nucleation probability $P$ was calculated as (Lazaridis et al., 1992)

$P=1-\exp \left(-J_{\text {het }} \cdot t\right)$

with $t$ being the nucleation time. This is the time a particle spends in a control volume, i.e. the $\mathrm{x}$-length of the control volume divided by the $\mathrm{x}$-velocity of the flow. $J_{\text {het }}$ is the nucleation rate per seed particle and unit time. Heterogeneous nucleation probabilities were calculated for each control volume based on the local thermodynamic properties resulting from the coupled solution of Eqs. (1) to (4). Within the CPC, heterogeneous nucleation probability changes very rapidly between zero and 1.0 (see Fig. 2). Therefore, in the simulations, particles/droplets were assumed to be activated and able to grow due to vapour condensation, for values of heterogeneous nucleation probability $P \geq 0.5$.

\subsubsection{Vapour condensation}

The condensational growth of the nucleated (both homogeneous and heterogeneous) particles/droplets was modelled according to Barrett and Clement (1988) accounting for elevated droplet temperature due to the latent heat released.

$$
\frac{\partial m_{p, i}}{\partial t}=\frac{2 \pi d_{p}\left(S-S^{*}\right)}{\frac{R_{v} T\left(1-\omega_{v} \frac{M}{M_{v}}\right)}{D_{v} p_{v, e} f_{\text {mass }}}+\frac{L_{v}^{2} S^{*}}{R_{v} T^{2} k f_{\text {heat }}}}
$$

where $S$ and $S^{*}$ are the vapour saturations in the gas phase and at the particle-droplet surface, respectively, $d_{p}$ is the 
particle-droplet diameter, $R_{v}$ is the vapour specific gas constant, and $p_{v, e}$ is the equilibrium vapour pressure. $f_{\text {mass }}$ and $f_{\text {heat }}$ are the mass and thermal accommodation coefficients, respectively. All other symbols are defined as above.

As mentioned before, for solving the above fluid and particle dynamical equations FLUENT/FPM was utilized. Using FLUENT/FPM implies performing Eulerian simulations for both fluid and particle dynamics and consequently discretization of the calculation domain in a numerical grid. As the system features rotational symmetry, we used a twodimensional axisymmetric grid. Since the walls are vapour and heat sources, the grid is finer towards the walls. Additionally, the grid resolution is highest in the expected activation zone (i.e. the growth tube), and lower for inlet and outlet areas. Since the wick puts a little distance between saturator and growth tube and the flow, the aerosol flow does not experience the temperature step between saturator and growth tube as a jump. To account for this, the temperature at the walls of our model is set to grow linearly over a small space from saturator to growth tube temperature. Altogether, the model grid consists of 178000 cells.

The following assumptions concerning the particle size distribution were made and utilized in different modelling experiments:

- A unimodal moving monodisperse size distribution for investigating how counting efficiency is affected by heterogeneous nucleation processes.

- A uni- and bimodal moving monodisperse size distribution for examining how counting efficiency is affected by particle number concentration.

- A bimodal (moving monodisperse plus polydisperse mode) when investigating how counting is efficiency affected by nucleation.

It should be noted that the model, despite its complexity and the number of processes accounted for, still has to be considered half quantitative as it still features some important assumptions, which include issues related to the validity of classical homogeneous and heterogeneous nucleation theory, and saturation boundary condition at the saturator and growth tube walls. However, applying the model to understand the behaviour of the CPC 3785, will give important information and insight concerning the behaviour of this specific instrument and Condensation Particle Counters in general.

\section{Results}

For a better overview of the complex coupled fluid flow, heat/mass transfer, particle dynamical processes taking place inside a TSI water CPC model 3785 (WCPC), and the thermodynamic property fields resulting from these processes, Fig. 2 depicts contours of temperature, water vapour mass concentration, water vapour saturation, and heterogeneous nucleation probability in the saturator and growth tubes of the WCPC.

Figure 2 clearly shows that the effect of the heated wall propagates into the aerosol flow (upper left panel), and how the flow is humidified by the wetted walls (upper right panel). Modelled temperature and water vapour mass fraction are the highest at the wall and the lowest at the centre line, with both increasing towards the growth tube outlet. It should be noted, that at the outlet, the flow and the surrounding wall are not in equilibrium with respect to either temperature or water vapour mass fraction. The resulting water vapour saturation field features the lowest values at the wall and a local maximum at the growth tube centre line. The field in our calculations is similar to the results presented in Hering and Stolzenburg (2005), where they introduced this method of generating supersaturated conditions with respect to water vapour and subsequently applied it to a commercial instrument (Hering et al., 2005). The maximum supersaturation at the centre line in Hering and Stolzenburg (2005) is higher (2.96) whereas in our calculations, the maximum was 1.94 . This is a consequence of a lower temperature difference between the saturator and the growth tube in our calculations. However, in both approaches radial and axial profiles are similar.

Of special interest is the lower right panel of Fig. 2. It points out the narrow region (the region where, coming from the left, the colour changes from dark blue to red) in which heterogeneous nucleation actually takes place. This steep increase in the nucleation probability is a key issue in understanding the CPC counting efficiency behaviour.

\subsection{Heterogeneous nucleation}

In order to study the effect of heterogeneous nucleation on the counting efficiency of the WCPC, we modelled the behavior of monodisperse $\mathrm{Ag}$ particles inside the CPC 3785 treating the activation of these particles as heterogeneous nucleation of water vapour. For these investigations a unimodal moving monodisperse size distribution was assumed. Results are given in Fig. 3 which depicts counting efficiency as function of particle diameter for different contact angles including also experimental data from (Petäjä et al., 2006) (Fig. 3a), and cut-off diameter as function of growth tube temperature (Fig. 3b).

Assuming a contact angle of $43.5^{\circ}$, which is in the range suggested by Wagner et al. (2003) for silver, the comparison between the numerical and the experimental data (Fig. 3a) seems to be encouraging. However, Fig. $3 b$ reveals that the agreement is merely occurring by chance. As to be seen, assuming a contact angle of $43.5^{\circ}$ yields significant differences in the cut-off diameter for the growth tube temperatures smaller and larger than approximately $330 \mathrm{~K}$ (the intersection of the red and the blue curve). The shapes of the two curves differ significantly. Considering a smaller contact angel, e.g. $30^{\circ}$, shapes of the experimental and theoretical 


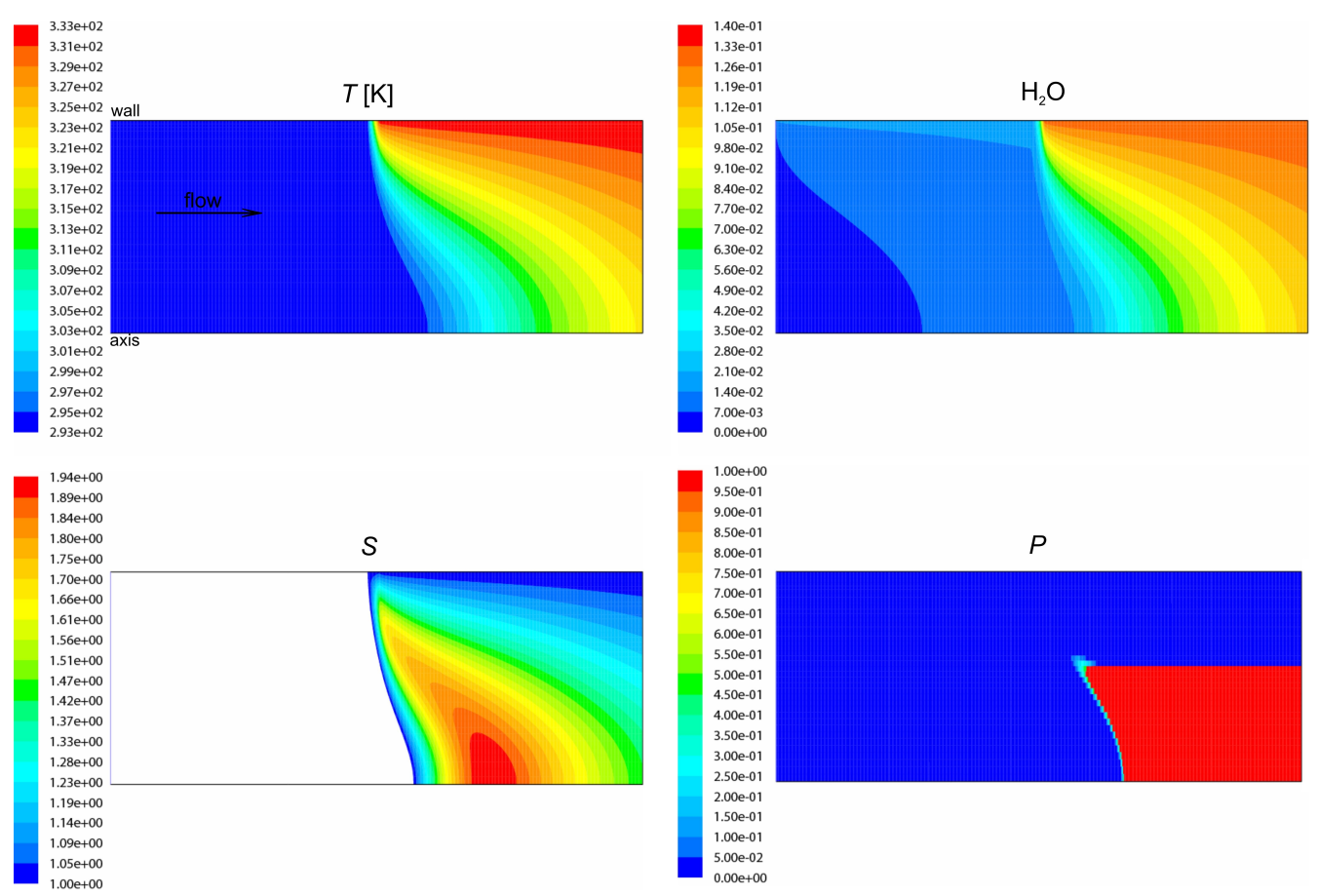

Fig. 2. Contours of temperature (upper left panel, $T[\mathrm{~K}]$ ), water vapour mass fraction (upper right panel, $\mathrm{H}_{2} \mathrm{O}$ ), water vapour saturation ratio (lower left panel, $S$ ), and heterogeneous nucleation probability for $6 \mathrm{~nm}$ seed particles (lower right panel, $P$ ) inside the TSI 3785 WCPC with a saturator temperature $T_{\mathrm{S}}=20^{\circ} \mathrm{C}$ and growth tube temperature $T_{\mathrm{gt}}=60^{\circ} \mathrm{C}$.

a

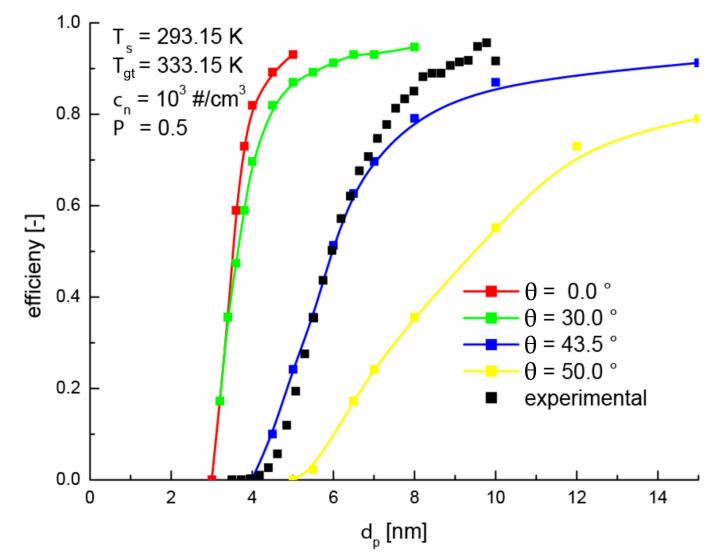

b

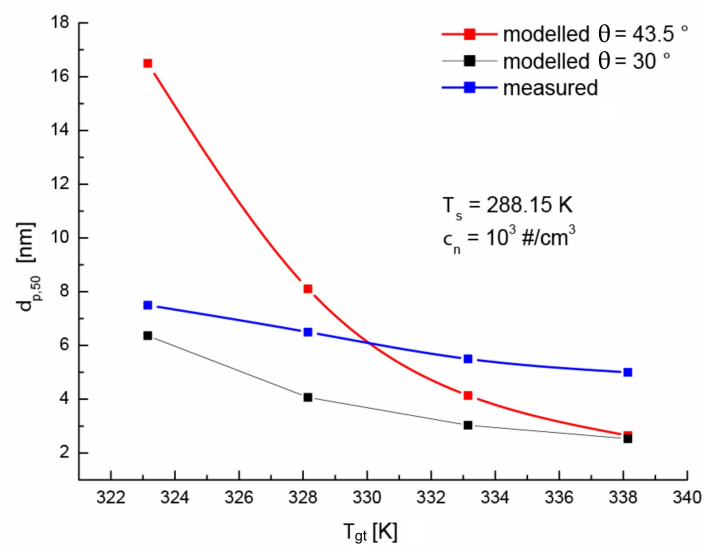

Fig. 3. CPC counting efficiency as a function of particle diameter (a) and cut-off diameter as function of growth tube temperature (b).

curves become similar, but the predicted cut-off size is too small. This suggests that besides the contact angle, also a correction to the kinetic term, i.e. an additional pre-factor in the heterogeneous nucleation rate, could be sufficient for matching the experimental and theoretical data.

However, determination of the actual contact angle and the corresponding pre-factor was not within the scope of the investigations presented here and will therefore be left for future research. From the above results it can be concluded that in the activation of Ag particles (and most likely that of other insoluble particles too) inside a CPC using water (most likely other substances too) as working fluid, the heterogeneous nucleation process has to be considered. Furthermore it should be noted that heterogeneous nucleation theory with only the contact angle as a free parameter is not able to consistently explain the experimental results.

\subsection{Effects of particle number concentration on the modelled counting efficiency}

To investigate the influences of particle number concentration on the WCPC counting efficiency, two scenarios were used. First, the response of the WCPC was modelled assuming a unimodal monodisperse (particle diameter $d_{p}=6 \mathrm{~nm}$ ) 
a

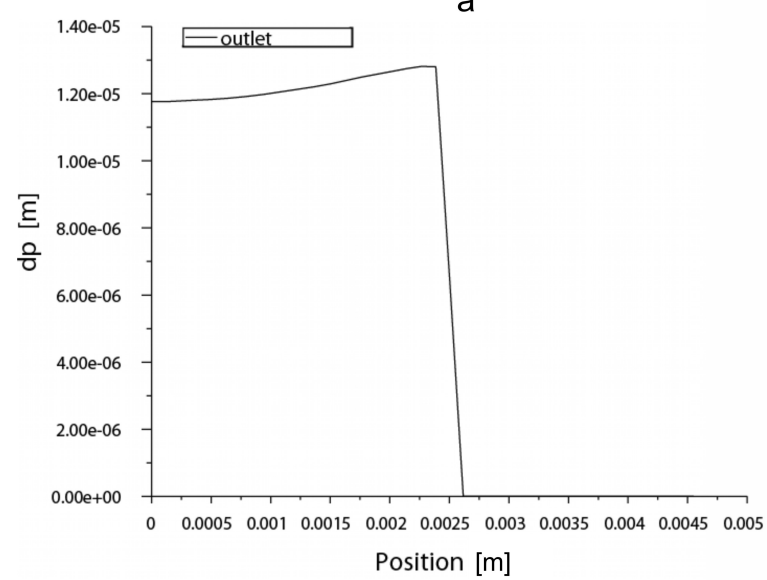

b

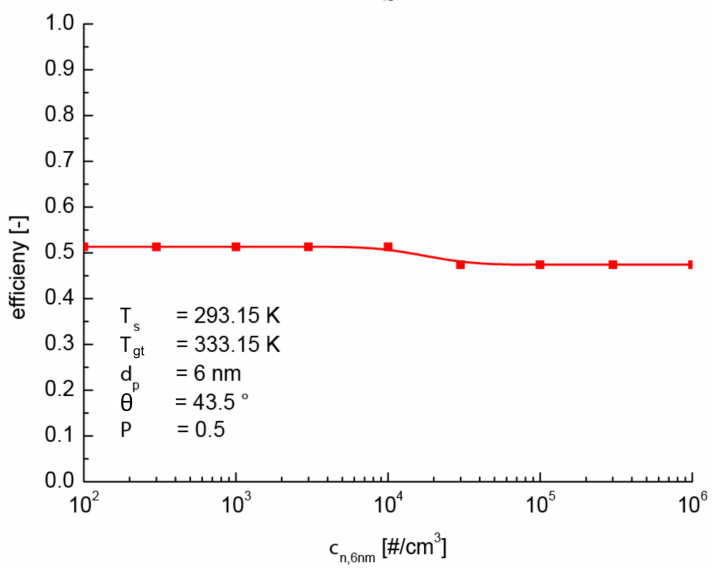

Fig. 4. Radial distribution of particle size at the growth tube outlet (a) and CPC counting efficiency as a function of inlet particle number concentration (b) assuming a unimodal inlet size distribution.

a

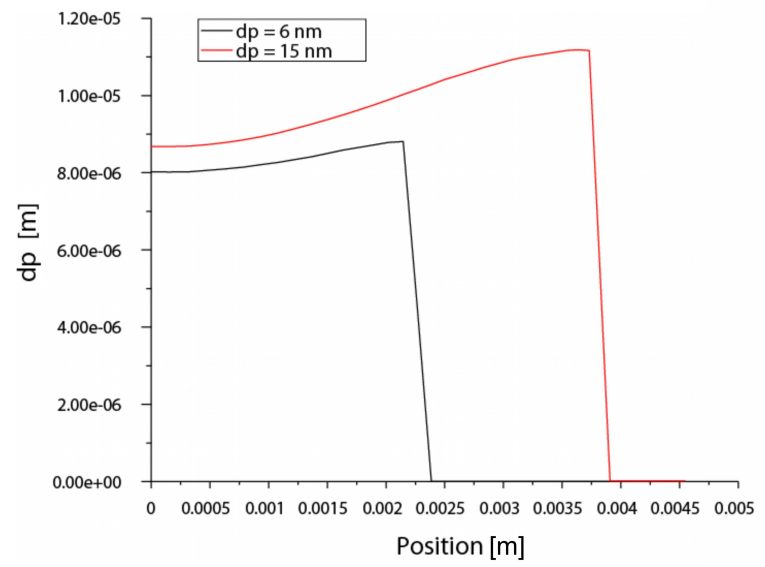

b

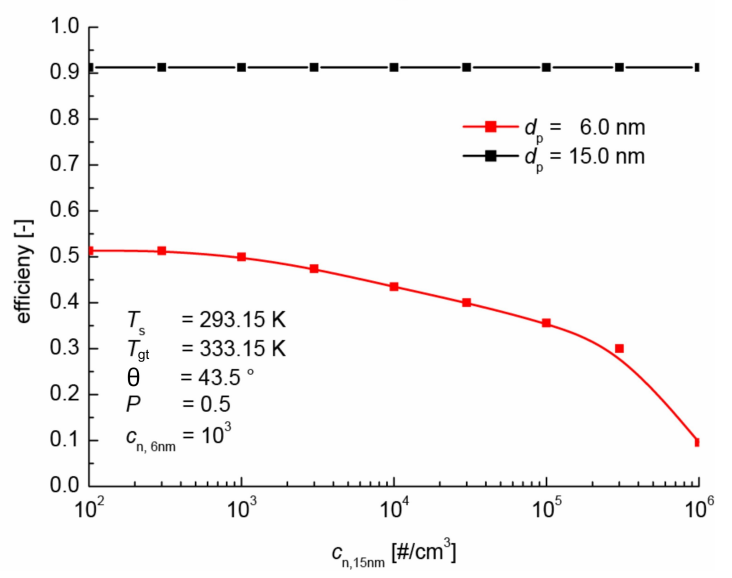

Fig. 5. Radial distribution of particle size at the growth tube outlet (a) and CPC counting efficiency as function of inlet particle number concentration (b) in a bi-modal modelling experiment.

size distribution, and subsequently a bimodal, consisting of two monodisperse populations with particle sizes of 6 and $15 \mathrm{~nm}$, respectively, were introduced to the modelling framework.

As can be seen from Fig. 4, the particle size at the growth tube outlet is almost constant (left panel), and the CPC counting efficiency is not dependent on the inlet number concentration for number concentrations as high as $10^{6} \mathrm{~cm}^{-3}$. This result, on first sight, is somewhat surprising but easy to understand looking at Fig. 2 (lower right panel). All the particles are activated in a narrow axial region, i.e. in the region where the heterogeneous nucleation probability jumps from zero to one, and consequently the activation itself is not much affected by vapour depletion. The particles at the growth tube outlet, however, become smaller (see also below) but even for a concentration of $10^{6} \mathrm{~cm}^{-3}$ their size is above the detection limit of the OPC which is here assumed to be one micrometer. In practice, the WCPC is capable of detecting particles in the single-particle, live-time corrected counting mode up to a concentration of $2 \times 10^{4} \mathrm{~cm}^{-3}$ and according to the manufacturer specifications, up to $10^{7} \mathrm{~cm}^{-3}$ in a photometric mode (TSI Inc, 2005). In other words, when counting monodisperse particles, reduced counting efficiency due to vapour depletion is hardly an issue at all.

Figure 5 depicts the radial distribution of particle size at the growth tube outlet (Fig. 5a) and CPC counting efficiency as function of inlet particle number concentration (Fig. 5b). In contrast to Fig. 4, now the results for a bimodal size distribution (initial particle sizes are 6 and $15 \mathrm{~nm}$, respectively) at the CPC inlet, are presented.

For both particle populations, Fig. 5a shows a characteristic behaviour: particle size increases with radial distance before dropping sharply off. The latter is caused by the fact that particles close to the walls are simply not activated. This can also be observed in Fig. 2, lower right panel. That particles off the center line grow larger has various reasons. First 


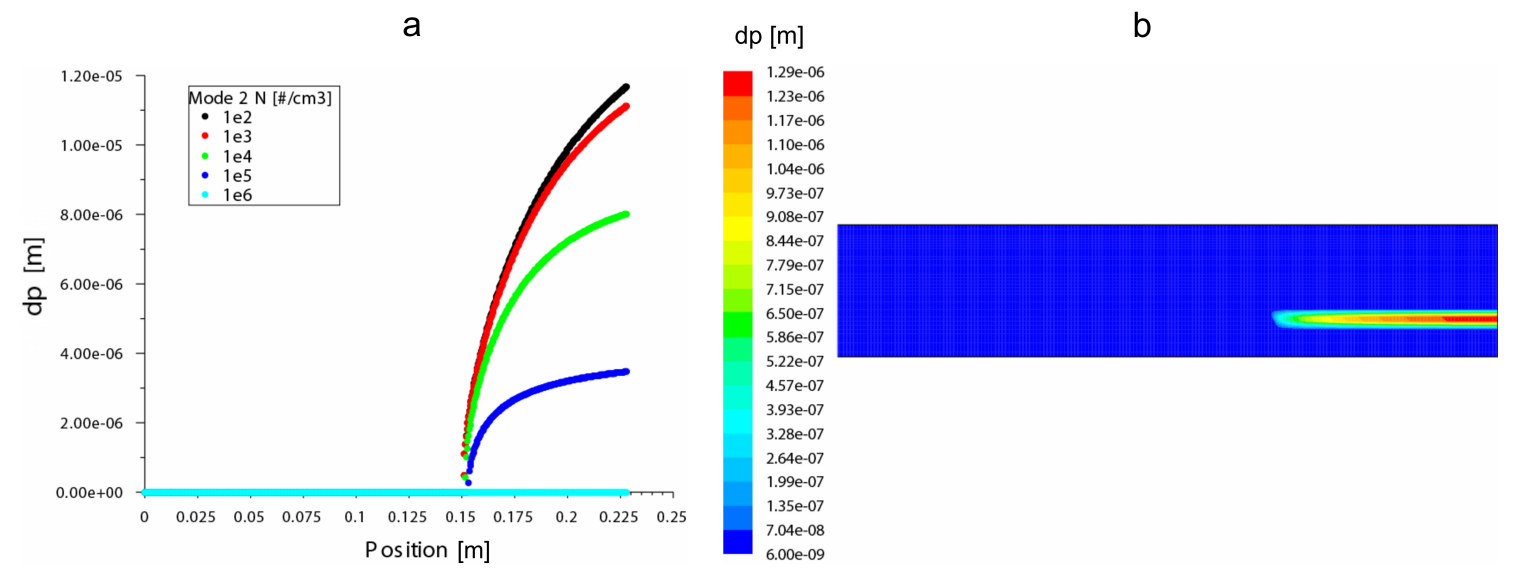

Fig. 6. Mode $2\left(d_{p, 2}=15 \mathrm{~nm}\right)$ axial particle diameter profiles for a bimodal particle size distribution $\left(a, T_{\mathrm{s}}=293.15 \mathrm{~K}, T_{\mathrm{gt}}=333.15 \mathrm{~K}\right.$, $\left.d_{p, 1}=6 \mathrm{~nm}, c_{n, 1}=10^{4} \# / \mathrm{cm}^{3}\right)$, and mode 1 particle diameter distribution $\left(b, T_{\mathrm{s}}=293.15 \mathrm{~K}, T_{\mathrm{gt}}=333.15 \mathrm{~K}, d_{p, 1}=6 \mathrm{~nm}, c_{n, 1}=10^{3} \# / \mathrm{cm} 3\right.$, $\left.d_{p, 2}=15 \mathrm{~nm}, c_{n, 2}=10^{5} \# / \mathrm{cm}^{3}\right)$.

of all, activation does not start on the center line as can be seen in the lower right panel of Fig. 2. This means that those particles off the center line have more time to grow. This is enhanced by the laminar flow profile implying that the farther away from the center, the slower the flow and thus particle movement. This again gives particles off the center line more time to grow. Both effects combined explain what Fig. 5a shows.

From Fig. 5a it becomes obvious that the two populations feature different grown sizes at the growth tube outlet. As suggested by intuition, particles with the initial size of $15 \mathrm{~nm}$, which activate at a smaller supersaturation, exhibit a larger diameter (around 10 micrometer) than the initially $6 \mathrm{~nm}$ particles (final size around 8 micrometer) which activate later and thus have less time to grow. Additionally, they might suffer from the vapour depletion caused by the larger particles.

The effect of vapour depletion by a larger particle population becomes more clearly visible when looking at the counting efficiencies. As to be seen from Fig. 5b, the counting efficiency for the $6 \mathrm{~nm}$ particles is a function of the concentration of the $15 \mathrm{~nm}$ particles reducing from $50 \%$ to $10 \%$ when changing the $15 \mathrm{~nm}$ particle concentration from $10^{3}$ to $10^{6} \mathrm{~cm}^{-3}$. The counting efficiency of the larger size particles is almost constant for the same reasons as discussed for the unimodal case.

Further insight can be gained by looking at Fig. 6 depicting particle mode 2 (initial diameter $15 \mathrm{~nm}$ ) diameter as a function of axial position inside the saturator and growth tube tubes (left panel) and a contour plot of mode 1 (initial diameter $6 \mathrm{~nm}$ ) particle diameter. It needs to be noted that Fig. 6a shows data only for the center line of the growth tube. The non-activation for the highest particle concentrations thus only tells us that particles on the center line do not activate. Off the center line, they may very well activate.
From Fig. 6a it is clearly seen that with increasing number concentration in mode 2 , the mode 2 particle diameter at the growth tube outlet is decreasing due to the particles competing for the vapour available. However, even for a concentration as high as $10^{5} \mathrm{~cm}^{-3}$, the particle size is larger than 2 micrometers, i.e. well above the detection limit of the CPC optics. Figure $6 \mathrm{~b}$ visualizes a rather interesting effect concerning the behaviour of the mode 1 (initial diameter $6 \mathrm{~nm}$ ) particle diameter. Due to the lack of vapour caused by the growth of the particles in the mode 2 , the activation and growth of the initially smaller particles is confined to a narrow off-center region.

Summarizing the results concerning the question of how counting efficiency is affected by particle number concentration it can be stated that for a monodisperse seed aerosol, particle number concentration does not affect the CPC counting efficiency, at least within certain limits. Of course counting efficiency will suffer if the particle number concentration is too big. However, this will only occur at almost unrealistically high number concentrations $\left(>10^{6} \mathrm{~cm}^{-3}\right)$. For a polydisperse aerosol, the number concentration of larger particles present can strongly affect the counting efficiency of the smaller particles, with the effect becoming noticeable at moderate concentrations $\left(>10^{3} \ldots 10^{4} \mathrm{~cm}^{-3}\right)$ of the larger particles.

\subsection{Effects of homogeneous nucleation to the counting efficiency}

In order to study the effect of homogeneous nucleation processes on the counting efficiency of the WCPC, a bimodal distribution with a moving monodisperse seed particle mode and a polydisperse lognormal nucleation mode particle size distribution were introduced to the modelling framework. Results of these investigations are presented in Fig. 7 which shows total particle number flux (number of particles per unit 
a

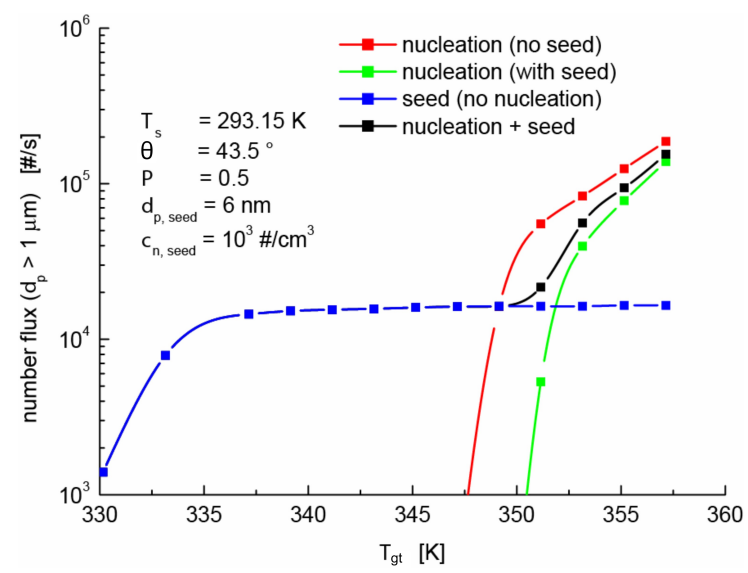

b

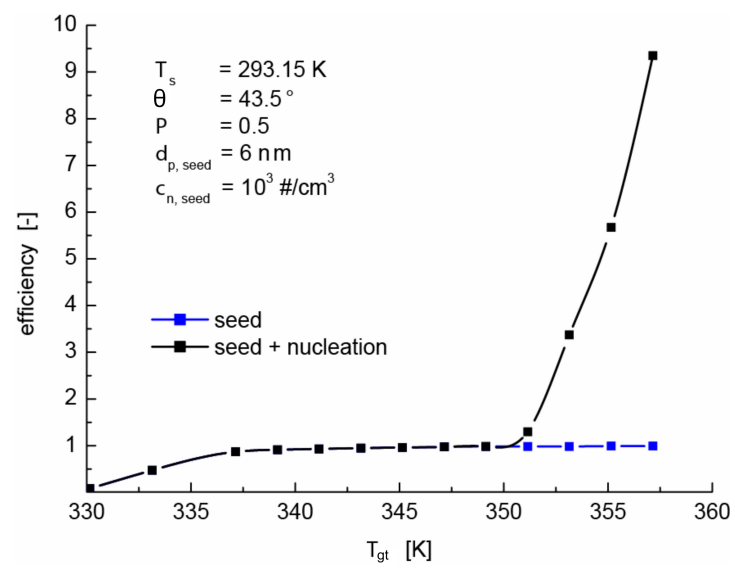

Fig. 7. Modelled particle number flux at the growth tube outlet (a), and counting efficiency as function of growth tube temperature (b).

time) out of the growth tube (Fig. 7a), and the corresponding $\mathrm{CPC}$ counting efficiency as function of growth tube temperature (Fig. 7b).

Looking at Fig. 7a, different curves can be distinguished. The blue curve, labelled "seed (no nucleation)", was determined neglecting homogeneous nucleation processes. This curve features an initial increase and converges to the number flux corresponding to a counting efficiency of unity (right panel). For calculating the red curve in Fig. 7a, labelled "nucleation (no seed)", only homogeneous nucleation was accounted for, i.e. possible influences of existing seed particles were neglected. From this curve it can be seen that without seed particles, homogeneous nucleation becomes important for growth tube temperatures larger than approximately $75^{\circ} \mathrm{C}$. This temperature is moved to slightly higher values (approximately $79^{\circ} \mathrm{C}$ ), when the influences of pre-existing seed particles are considered, as to be seen examining the green curve labelled "nucleation (with seed)". The "real" number flux at the growth tube outlet is represented by the black curve labelled "nucleation + seed" which features a significant increase for growth tube temperatures larger than $80^{\circ} \mathrm{C}$. The CPC counting efficiency (Fig. 7b) increases accordingly. Consequently it can be stated that homogeneous nucleation processes may result in an increase in the CPC counting efficiency yielding values of up to 10 . Thus, with a large temperature difference between the saturator and the growth tube, the modelling suggests a large contribution to the measured particle concentration due to homogeneous nucleation of water vapour inside the WCPC.

\section{Conclusions}

The key parameters determining the detection efficiency of a Condensation Particle Counter are the level of supersaturation and both the physico-chemical properties of the working fluid and the sampled particles (e.g. Stolzenburg and
McMurry, 1991; Kulmala et al., 2007; Iida et al., 2009). In the case of insoluble particles, heterogeneous nucleation describes the formation of nuclei onto the pre-existing particles (Lazaridis et al., 1992). In this work the influences of various effects on the counting efficiency of a TSI 3785 CPC were investigated theoretically. For describing the coupled fluid and particle dynamical processes taking place inside the CPC, the Computational Fluid Dynamics Code FLUENT (Fluent, 2005) together with the Fine Particle Model (FPM) (particle dynamics, 2005) were used.

Summarizing the key results, the investigations showed that heterogeneous nucleation processes should be considered in modelling the activation and the growth of silver particles inside the WCPC. The heterogeneous nucleation theory was not, however, able to consistently explain all of the experimental results. For a monodisperse silver seed aerosol, the particle number concentration did not affect the CPC counting efficiency in the concentration range below $10^{6} \mathrm{~cm}^{-3}$. For a polydisperse aerosol, the number concentration of the larger particles has an effect on the counting efficiency of the smaller particles due to vapour depletion. In addition, in case of large supersaturations inside the WCPC, homogeneous nucleation of water vapour can produce a significant erroneous signal due to particle production inside the $\mathrm{CPC}$ and thus lead to large changes in the perceived counting efficiency.

The results confirm that the counting efficiencies of CPCs are dependent on both particle and vapour chemical composition. Consequently, at least for nanometer particles, there is neither a unique cut-off diameter nor a single counting efficiency. When considering the measurements of total particle number concentration in e.g. an atmospheric polydisperse aerosol particle population, the results imply that the counting efficiency of small, freshly nucleated particles, might be a function of the number and size of the pre-existing Aitken and accumulation mode particles, resulting in biased total 
number concentrations. This should be kept in mind when interpreting the atmospheric total number concentration data in cases when sub-10 $\mathrm{nm}$ particles are present in large quantities. Scenarios to consider include for example an urban setting with high concentrations of combustion particles or a rural area that experiences a new particle formation event.

Edited by: J. Gumbel

\section{References}

Barrett, J. C. and Clement, C. F.: Growth rates for liquid drops, J. Aerosol Sci., 19, 223-242, 1988.

Fletcher, N. H.: Size effect in heterogeneous nucleation. J. Chem. Phys. 29, 572-576, 1958.

Fluent: FLUENT 6.2 User's Guide, Fluent Inc., Lebanon NY, USA, 2005.

Girshick, S. L., Chiu, C.-P., and McMurry, P. H.: Time-dependent aerosol models and homogeneous nucleation rates, Aerosol Sci. Technol., 13, 465-477, 1990.

Hering, S. V. and Stolzenburg, M. R.: A method for particle size amplification by water condensation in a laminar, thermally diffusive flow, Aerosol Sci. Technol., 39, 428-436, 2005.

Hering, S. V., Stolzenburg, M. R., Quant, F. R., Oberreit, D. R., and Keady, P. B.: A laminar-flow, water-based-condensation particle counter (WCPC), Aerosol Sci. Technol., 39, 659-672, 2005.

Iida, K., Stolzenburg, M. R., and McMurry, P. H.: Effect of working fluid on sub-2 $\mathrm{nm}$ particle detection with a laminar flow ultrafine condensation particle counter, Aerosol Sci. Technol. 43, 81-96, 2009.

Kulmala, M., Kerminen, V.-M., Anttila, T., Laaksonen, A., and O'Dowd, C. D.: Organic aerosol formation via sulphate cluster activation, J. Geophys. Res., 109, D04205, doi:10.1029/2003JD003961, 2004.

Kulmala, M., Mordas, G., Petäjä, T., Grönholm, T., Aalto, P. P., Vehkamäki, H., Hienola, A. I., Herrmann, E., Sipilä, M., Riipinen, I., Manninen, H., Hämeri, K., Stratmann, F. , Bilde, M., Winkler, P. M., Birmili, W., and Wagner, P. E.: The condensation particle counter battery (CPCB): a new tool to investigate the activation properties of nanoparticles, J. Aerosol Sci., 38, 289-304, 2007.
Kürten, A., Curtius, J., Nillius, B., and Borrmann, S.: Characterization of an automated, water-based expansion condensation nucleus counter for ultrafine particles, Aerosol Sci. Techol. 39, 1174-1183, 2005.

Lazaridis, M., Kulmala, M., and Laaksonen, A.: Binary heterogeneous nucleation of a water-sulphuric acid system: The effect of hydrate interaction, J. Aerosol Sci., 22, 823-830, 1991.

Lazaridis, M., Kulmala, M., and Gorbunov, B.: Heterogeneous Nucleation at a non- uniform surface, J. Aerosol Sci., 23, 457-466, 1992.

McMurry, P. H.: A review of atmospheric aerosol measurements, Atmos. Environ., 34(12-14), 1959-1999, 2000.

Particle dynamics: Fine Particle Model (FPM) for FLUENT, Particle Dynamics GmbH, Leipzig, Germany (http://www. particle-dynamics.de/), 2005.

Petäjä, T., Mordas, G., Manninen, H., Aalto, P. P., Hämeri, K., and Kulmala, M.: Detection efficiency of a water-based TSI condensation particle counter 3785, Aerosol Sci. Technol., 40, 10901097, 2006.

Saros, M. T., Weber, R. J., Marti, J. J., and McMurry, P. H.: Ultrafine aerosol measurement using a condensation nucleus counter with pulse height analysis, Aerosol Sci. Technol., 25, 200-213, 1996.

Sem, G. J.: Design and Performance Characteristics of Three Continuous-Flow Condensation Particle Counters: A Summary, Atmos. Res., 62, 267-294, 2002.

Sgro, L. A. and Fernandez de la Mora, J.: A simple turbulent mixing $\mathrm{CNC}$ for charged particle detection down to $1.2 \mathrm{~nm}$. Aerosol Sci. Technol. 38, 1-11, 2004.

Stolzenburg, M. R. and McMurry, P. H.: An Ultrafine Aerosol Condensation Nucleus Counter, Aerosol Sci. Technol., 14, 48-65, 1991.

TSI: Model 3785 Water-based Condensation Particle Counter: Operation and Service Manual, TSI Inc., Shoreview MN, USA, 2005.

Wagner, P. E., Kaller, D., Vrtala, A., Lauri, A., Kulmala, M., and Laaksonen, A.: Nucleation probability in binary heterogeneous nucleation of water- $n$-propanol vapor mixtures on insoluble and soluble nanoparticles. Phys. Rev. E, 67, 021605.1-021605.12, 2003. 